This is the peer reviewed version of the following article Chem. Eur. J. 2015, 21 (16), 6115., which has been published in final form at [10.1002/chem.201406334]. This article may be used for non-commercial purposes in accordance with Wiley Terms and Conditions for Self-Archiving.

\title{
Al(III) Catalyzed Formation of Poly(limonene)carbonate: DFT Analysis of the Origin of Stereoregularity
}

\author{
Leticia Peña Carrodeguas, ${ }^{[\mathrm{a}]}$ Fernando Castro-Gómez, ${ }^{[\mathrm{a}]}$ Joan González-Fabra, ${ }^{[\mathrm{a}]}$ Carles Bo ${ }^{*[\mathrm{a}, \mathrm{b}]}$ and \\ Arjan W. Kleij ${ }^{\star[a, c]}$
}
[a] L. Peña Carrodeguas, Dr. F. Castro-Gómez, J. González-Fabra, Prof. Dr. C. Bo, Prof. Dr. A. W. Kleij Institute of Chemical Research of Catalonia (ICIQ), Av. Països Catalans 16, 43007 Tarragona, Spain E-mail: akleij@iciq.es
[b] Prof. Dr. C. Bo Departament de Química Física i Inorgànica, Universitat Rovira i Virgili, Marcel-lí Domingo s/n, 43007 Tarragona, Spain
[c] Prof. Dr. A. W. Kleij Catalan Institute of Research and Advanced Studies (ICREA), Pg Lluís Companys 23, 08010 Barcelona, Spain
Supporting information for this article is given via a link at the end of the document.

\begin{abstract}
Amino-triphenolate derived $\mathrm{Al}(\mathrm{III})$ complexes combined with suitable nucleophiles have been investigated as binary catalysts for the coupling of limonene oxide and carbon dioxide to afford alternating polycarbonates. These catalysts are able to produce stereo-regular, perfectly alternating trans-polymers from cis-limonene oxide, whereas the pure trans isomer and cis/trans mixture give rise to lower degrees of stereo-regularity. The best $\mathrm{Al}(\mathrm{III})$ catalyst shows the potential to mediate the conversion of both stereo-isomers of limonene oxide with high conversion levels of up to $71 \%$ under neat conditions indicating a high robustness and atom-efficiency of this catalytic process. Computational studies have revealed unique features of the binary catalyst system among which is the preferred nucleophilic attack on the quaternary carbon centre in the limonene oxide substrate.
\end{abstract}

\section{Introduction}

Currently there is a high demand for chemical processes that enable the conversion of renewable feed stocks into value-added chemicals as to increase the overall sustainability of our societies. ${ }^{[1]}$ In this regard, the use of carbon dioxide as a carbon resource has attracted much interest during the last decade and substantial progress has been made to use this synthon in organic synthesis. ${ }^{[2]}$ The co-polymerization of epoxides and $\mathrm{CO}_{2}$ is a successful and relevant example of using a renewable carbon feed stock and converting it into a material of widespread commercial and academic interest. ${ }^{[3]}$ However, chemical processes leading to such $\mathrm{CO}_{2}$-based materials still depend on petroleum-based feed stocks despite the impressive advancements made in this area using various types of epoxide monomers. ${ }^{[4,5]}$

The use of bio-renewable based monomers such as limo-nene oxide has received far less attention, and we are aware of only a few reports dealing with the successful co-polymerization of this monomer with $\mathrm{CO}_{2} \cdot{ }^{[5]}(R)$-Limonene is a naturally occurring terpene that is available in large amounts, and the epoxidized form is commercially available at low cost as a mixture of cis and trans isomers (Figure 1; $\mathbf{A}$ and $\mathbf{B}$ ). Its structural resemblance to cyclohexene oxide (the most widely used epoxide in copolymerization reactions with $\mathrm{CO}_{2}$ ) makes it thus an ideal target to provide a cost-effective, bio-based polycarbonate from a renewable feed stock. Though, some important challenges remain to be solved: limonene oxide represents an internal, tri-substituted epoxide monomer and the kinetic barrier for its activation is significantly higher than for terminal epoxides. ${ }^{[6]}$ There are only relative few reports describing the efficient conversion of internal epoxides into their respective organic carbonates, ${ }^{[7-11]}$ and the development of (more) powerful catalytic systems ${ }^{[7]}$ is thus of vital importance to solve these synthetically more challenging preparations that involve such monomers. 


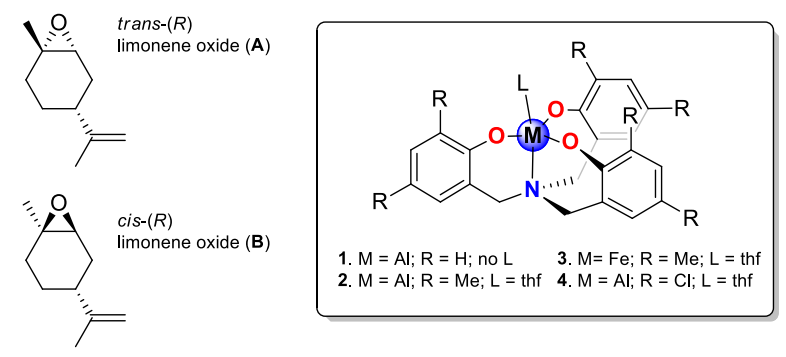

Figure 1. Schematic structures of the amino-trisphenolate metal complexes 1-4 used in this work, and cis and trans $(R)$-limonene oxide.

We recently developed $\mathrm{Fe}(\mathrm{III})^{[8]}$ and $\mathrm{Al}(\mathrm{III})^{[9,11]}$ amino-triphenolate complexes 1-4 (Figure 1) that show high efficiency in the formation of functional organic carbonates from both terminal and internal epoxides. Further to this, we also found that these complexes have the intrinsic ability to switch between penta- and hexa-coordination, ${ }^{[1]}$ a feature that may be useful in the creation of flexible coordination behavior around the metal ion in the presence of sterically more demanding substrates. ${ }^{[12]}$

In this work we report on a rare case of a catalytic system able to mediate the efficient and stereo-selective formation of poly(limonene)carbonate, a tri-substituted oxirane monomer. Further to this, high conversion of the monomer limonene oxide can be attained (up to $71 \%$ ) with the cis isomer reacting significantly faster than the trans analogue. The rationale behind these observed reactivity patterns has been investigated in detail using density functional theory (DFT) providing unique insight into the operative modus of the catalyst system. Catalysts as the one reported herein pave the way for the conversion of other naturally occurring, renewable compounds into valuable chemicals including biopolymers that are derived from epoxide/ $\mathrm{CO}_{2} \mathrm{couplings}$. The development of such bio-based polymers may give useful alternatives for existing, environmentally less attractive and potentially toxic copolymers, of which bis-phenol A (BPA) based ones are most prominent.

\section{Results and Discussion}

\section{Screening studies}

Our previous results using amino-triphenolate complexes as catalysts for poly(cyclohexene)carbonate synthesis ${ }^{[12]}$ prompted us to evaluate their catalytic efficiencies in the co-polymerization of a commercially available mixture of cis/trans (40:60 by GC) (R)limonene oxide (see Table 1). Various combinations of catalysts (1-4; Scheme 1) and co-catalysts (nucleophiles abbreviated as $\mathbf{C l}$, $\mathrm{Br}$ and I) and their relative ratios were probed in a screening phase. At $42^{\circ} \mathrm{C}$ complex 1 (entry 1) exhibited no catalytic activity, whereas complex 2 (entry 2) gave a reason-able conversion of $20 \%$ furnishing a copolymer with high chemo-selectivity $\left(\mathrm{CO}_{2}\right.$ linkages $>99 \%$ ) and high trans incorporation (78\%). In contrast to the $\mathrm{Zn}(\mathrm{BDI})$ catalyst reported by Coates ${ }^{[5 \mathrm{a}]} \mathbf{2}$ shows lower activity but does maintain activity throughout a long period of time (entries 3-8). The presence of the Al-complex is a requisite for the synthesis of poly(limonene)carbonate as the use of co-catalyst alone $(P P N C l=C l$; entry 9$)$ does not lead to any observable conversion.

In general, Al-complex 2 proved to be the most efficient mediator of the copolymerization reaction and co-catalyst $\mathrm{Cl}(\mathrm{PPNCl})$ and $\mathrm{Br}$ (PPNBr) the best co-catalysts with the former one leading to a higher percentage of trans units in the polymer product; (co)catalyst loadings required for efficient catalysis were $0.5-1.0 \mathrm{~mol} \%$. Longer reaction times provided higher conversion levels, but increasing temperatures (entries 16 and 17) had no favorable effect on the activity/selectivity. ${ }^{[13]}$ As the reaction proceeds with time (entries 3-6) it can be noted that the amount of trans units in the polymer product stays stabile around $70 \%$. This result suggests that both trans- $(A)$ and cis- $(R)$-limonene oxide $(B)$ are converted by binary catalyst system $2 / \mathbf{C I}$. Interestingly, the ${ }^{1} \mathrm{H}$ NMR spectra of the isolated polymer samples display three separate peaks $(\delta=5.05,5.08$ and $5.12 \mathrm{ppm}$, respectively: see Figure 2 and the Supporting Information for more details) for the $\mathrm{CH}$ groups located near the carbonate linkages (colored blue in the scheme of Table 1). The peaks at 5.05 and $5.12 \mathrm{ppm}$ were previously assigned to different regio-related trans units in the poly(limonene)carbonate (Figure above Table 1$),{ }^{5}$ whereas the signal at $5.08 \mathrm{ppm}$ was tentatively assigned to the presence of cis units (Figure 2).

This screening study shows that aminotriphenolate complex $\mathbf{2}$ in combination with a suitable nucleophile additive (PPNCl) affords active and robust catalyst systems for limonene oxide $/ \mathrm{CO}_{2}$ couplings with high selectivity for the copolymer. 


Table 1. Co-polymerization of $(R)$-limonene oxide and $\mathrm{CO}_{2}$ using complexes
$1-4$ and various nucleophilic PPN-based co-catalysts.
ale

[a] Conditions: cis/trans $(R)$-limonene oxide $(1.0 \mathrm{~g}, 6.57 \mathrm{mmol})$, metal complex (quantity indicated), co-catalyst (quantity indicated), 10 bar initial $\mathrm{CO}_{2}$ pressure, $30 \mathrm{~mL}$ autoclave, neat. [b] Internal temperature inside the autoclave. [c] Determined by ${ }^{1} \mathrm{H}$ NMR of the crude reaction mixture with amount of carbonate linkages $>99 \%$. [d] Total \% trans in co-polymer determined by ${ }^{1} \mathrm{H}$ NMR using signal integration, see Supporting Information for more details.

\section{Optimization of the Copolymerization Reaction.}

In order to get a better understanding of the origin of the presence of trans and cis units in the isolated poly(limonene)carbonates, we then used pure trans and cis $(R)$-limonene oxide and investigated their behavior separately in the copolymerization with $\mathrm{CO}_{2}$, and compared the results with those obtained for the commercial mixture of cis/trans limonene oxide (Table 2). 

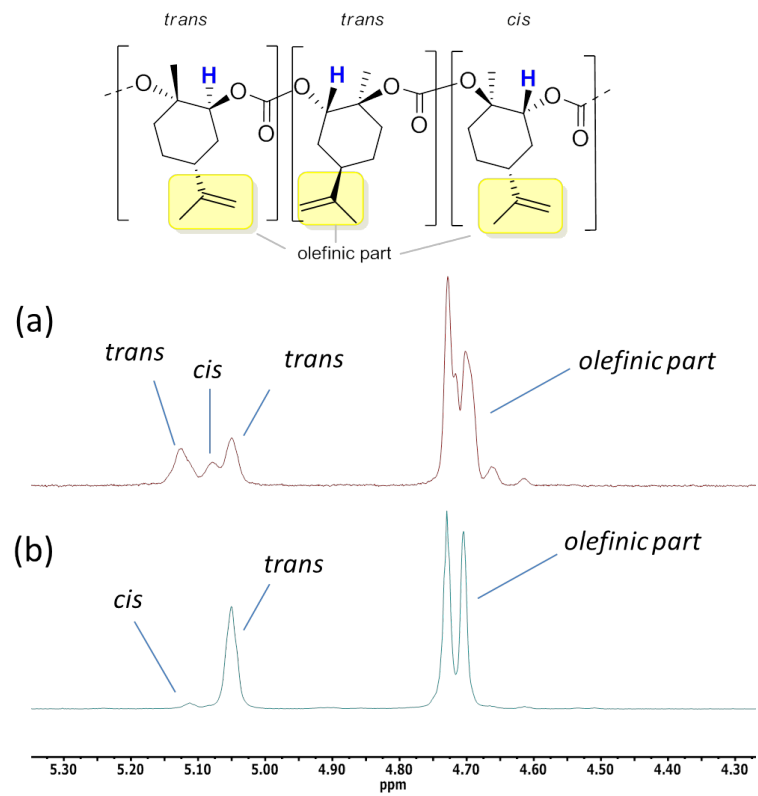

Figure 2. (a) Amplification (4.30-5.30 ppm) of a typical ${ }^{1} \mathrm{H}$ NMR spectrum of an isolated sample of poly(limonene)carbonate containing two types of trans units as well as one cis unit. Conditions: catalyst $2(1.0 \mathrm{~mol} \%), \mathrm{PPNCl}(0.5 \mathrm{~mol} \%), 42^{\circ} \mathrm{C}, 18 \mathrm{~h}, p\left(\mathrm{CO}_{2}\right)=10$ bar, mixture of $\mathbf{A}$ and $\mathbf{B}$ as substrate; (b) Comparative NMR trace for a copolymer obtained from pure cis limonene oxide, for conditions see entry 8 , Table 2 . Note that the peaks at $\delta=5.05,5.08$ and 5.13 ppm relate to the blue-coloured H's in the polymer figure. Further details are provided in the Supporting Information, Figures S4-S22.

The copolymerization of a commercial mixture of trans and cis limonene oxide $(\mathbf{A} / \mathbf{B})$ with $\mathrm{CO}_{2}$ in a conventional autoclave reactor furnishes poly(limonene)carbonate of relatively low molecular weight (entries $1-3 ; M_{\mathrm{n}}$ around $3.0 \mathrm{Kg} \cdot \mathrm{mol}^{-1}$ ) and the total reaction time seems to have little effect on the polymer properties (cf, entries 1-3 and 5-6), although some depolymerization of the formed polycarbonate cannot be ruled out completely. The total amount of trans units in the formed polycarbonate was followed in time and showed a stable progress in time amounting to about $70-75 \%$.

Interestingly, when more strict anhydrous conditions were used (entries 4-6; Fischer-Porter reactor, see Supporting Information, Figures S2 and S3) the molecular weight values increased up to $6.7 \mathrm{Kg} \cdot \mathrm{mol}-1$, indicating that chain transfer reactions by trace amounts of water are likely responsible for this difference. ${ }^{[14]}$ This assumption was supported by end-group inspection of the MALDITOF spectra recorded for these polymers (Supporting Information, Figures S23-S26). In all cases where the commercial mixture A/B of limonene oxide was used as substrate, the stereo-regularity of the produced copolymer was similar. Then, pure trans (A) and cis (B) limonene oxide were probed under similar polymerization conditions (entries 7-13) to assess whether the type of substrate has any influence.

\begin{tabular}{|c|c|c|c|c|c|c|c|}
\hline Entry & $S$ & $\begin{array}{c}\mathbf{C l} \\
{[\mathrm{mol} \%]}\end{array}$ & $\begin{array}{c}\mathrm{t} \\
\text { (h) }\end{array}$ & $\begin{array}{l}\text { Conv } \\
{[\%]^{[b]}}\end{array}$ & $\begin{array}{c}\text { Cis/Trans } \\
{\left[_{\%}\right]^{[c]}}\end{array}$ & $\underset{[\mathrm{d}, \mathrm{e}]}{M_{\mathrm{n}}}$ & $\underset{[d]}{M_{w} / M_{n}}$ \\
\hline 1 & $A / B$ & 0.5 & 12 & 37 & $25: 75$ & 2.9 & 1.33 \\
\hline 2 & $A / B$ & 0.5 & 24 & 47 & $24: 74$ & 3.1 & 1.38 \\
\hline 3 & $A / B$ & 0.5 & 48 & 60 & $30: 70$ & 2.4 & 1.49 \\
\hline $4^{[\mathrm{ff}]}$ & $A / B$ & 0.25 & 48 & 53 & 24:76 & 6.7 & 1.55 \\
\hline $5^{[\mathrm{[f]}}$ & $A / B$ & 0.5 & 24 & 31 & $25: 75$ & 5.2 & 1.42 \\
\hline $6^{[\text {[f] }}$ & $A / B$ & 0.5 & 87 & 54 & $26: 74$ & 5.5 & 1.47 \\
\hline
\end{tabular}




\begin{tabular}{|c|c|c|c|c|c|c|c|}
\hline 7 & A & 0.5 & 60 & 59 & $27: 73$ & 3.1 & 1.28 \\
\hline 8 & B & 0.5 & 24 & 67 & $7: 93$ & 7.0 & 1.32 \\
\hline $9^{[f]}$ & B & 0.5 & 24 & 71 & $2: 98$ & 5.9 & 1.40 \\
\hline $10^{[\mathrm{ff}}$ & B & 0.25 & 48 & 53 & 9:91 & 5.8 & 1.43 \\
\hline $11^{\text {[f] }}$ & B & 0.5 & 8 & 27 & 4:96 & 4.8 & 1.35 \\
\hline $12^{[f, g]}$ & B & 0.5 & 24 & 54 & $5: 95$ & 9.1 & 1.48 \\
\hline $13^{[f, 9]}$ & B & 0.5 & 24 & 49 & $4: 96$ & 10.6 & 1.43 \\
\hline $14^{[\mathrm{h}]}$ & $A / B$ & 3.0 & 34 & 42 & $>95^{i}$ & n.a. & n.a \\
\hline $15^{h}$ & A & 3.0 & 64 & 29 & $>95^{i}$ & n.a. & n.a \\
\hline $16^{h}$ & B & 3.0 & 64 & 3 & n.d. ${ }^{i}$ & n.a. & n.a \\
\hline
\end{tabular}

[a] Conditions: cis/trans, cis or trans $(R)$-limonene oxide $(1.0 \mathrm{~g}, 6.57 \mathrm{mmol})$ Al-complex 2 (1 mol\%), co-catalyst (quantity indicated in mol\%), 10 bar initial $\mathrm{CO}_{2}$ pressure, $30 \mathrm{~mL}$ autoclave, $42^{\circ} \mathrm{C}$, neat; $\mathrm{S}$ stands for the type of substrate. [b] Determined by ${ }^{1} \mathrm{H}$ NMR of the crude reaction mixture with amount of carbonate linkages $>99 \%$. [c] Total \% trans in co-polymer determined by ${ }^{1} \mathrm{H}$ NMR using signal integration, see Supporting Information for more details. [d] Determined by GPC, see Supporting Information for details. [e] In Kg. mol ${ }^{-1}$. [f] Reaction carried out in a Fischer-Porter reactor at 5 bar $/ 45^{\circ} \mathrm{C}$. [g] Different stirring technique applied (see Supporting Information). [h] Only co-catalyst $\mathrm{PPN}-\mathrm{Cl}$ used at $90^{\circ} \mathrm{C}$; only cyclic limonene carbonate observed. N.a. $=$ not applicable, n.d. = not determined.

Remarkably, whereas the use pure trans limonene oxide A (entry 7) results in the formation of a low molecular weight copolymer, the use of the pure cis isomer B gives substantially higher molecular weight material (entry 8 ) and its conversion is significantly faster. Moreover, the resultant copolymer based on pure B shows a higher degree of stereo-regularity ( $92 \%$ trans units, one type) compared with the copolymer based on pure trans A ( $73 \%$ trans units). This implies that the coupling of pure cis limonene oxide is more stereoselective and suggests that nucleophilic attack of the chloride is surprisingly favored on the most substituted carbon center of the oxirane unit in $\mathbf{B}$. The use of more strict anhydrous conditions failed to give improved polymer properties when using pure $\mathbf{B}$ as substrate (entries 9-11) despite varying both the co-catalyst concentration and reaction time. However, when a different stirring technique (Figure S3) ${ }^{[15]}$ was applied in the reactor (entries 12 and 13), improved molecular weights of the resultant copolymers could be achieved up to an appreciable $10.6 \mathrm{Kg} \cdot \mathrm{mol}-1$ (Figure S30) while maintaining similar polydispersities $\left(M_{\mathrm{w}} / M_{\mathrm{n}}\right.$ values) around 1.4 . Finally, attempts to convert limonene oxide (A/B mixture of isomers or pure isomers, entries 14-16) in the absence of catalyst 2 were done. The highest conversion was achieved with the commercial mixture of limonene oxide (at $\left.90^{\circ} \mathrm{C}\right)$, whereas the use of pure $\mathbf{A}$ $(29 \%)$ and pure B gave much lower conversion. In these latter reactions full selectivity for the cyclic limonene carbonate was noted (Figures S21, S22 and S32), emphasizing the crucial role of catalyst 2 to form the copolymer under much milder conditions.

The properties of the obtained poly(limonene)carbonates were further assessed by TGA and DSC analysis. ${ }^{[16]}$ The copolymers of lower molecular weight (e.g., entry 9, Table 2, cis monomer B used; $M_{\mathrm{n}}=5.9 \mathrm{Kg} \cdot \mathrm{mol}-1, M_{\mathrm{w}} / M_{\mathrm{n}}=1.40$; Figure S33) shows an onset temperature for decomposition around $180^{\circ} \mathrm{C}\left(T_{a}=241^{\circ} \mathrm{C}\right)$ measured by TGA, whereas higher molecular weight samples (cf., entries 12 and 13; Figure S34) display lower midpoint temperatures $T_{\mathrm{a}}$ of 204 and $199^{\circ} \mathrm{C}$, respectively. As for glass transition behavior, the $T_{\mathrm{g}}$ values among the polymer samples derived from a mixture of isomers A/B (entry 5, Table 2; Figure S35) or pure B (entry 9 , Table 2; Figure S36) both isolated after $24 \mathrm{~h}$ using similar reactions conditions were rather similar $\left(T_{\mathrm{g}}=78\right.$ and $81^{\circ} \mathrm{C}$, respectively). The highest molecular weight sample from entry 13 showed the highest $T_{\mathrm{g}}$ value around $112^{\circ} \mathrm{C}$ (Figure S38) comparable to what was found previously by Coates et al. for the poly(limonene)carbonate derived from the trans substrate $\mathbf{A}^{\text {[5a] }}$

Our results contrast in some aspects with those reported by Coates et al. ${ }^{[5 a]}$ in the sense that (1) lower activities are noted for the binary catalyst $\mathbf{2} / \mathbf{C I},(2)$ in the presence of this latter binary catalyst system the cis-isomer of limonene oxide reacts significantly faster than the trans one, whereas for the Coates system this isomer could not be converted, and (3) the use of the cis-isomer $\mathbf{B}$ results in a stereo-regular, virtually all trans-regular copolymer whereas the Coates catalyst is selective towards stereo-regular poly(limonene)carbonate based on the conversion of trans isomer $\mathbf{A}$. In order to get more insight into the chemo- and stereo-selective features of the catalytic process, detailed computational studies (DFT) were carried out to evaluate the difference in reactivity observed for isomers $\mathbf{A}$ and $\mathbf{B}$, and the origin for the selective conversion of pure cis limonene oxide into trans poly(limonene)carbonate (vide infra). 


\section{Computational Studies.}

Considering a bimetallic mechanism, ${ }^{[17]}$ we further investigated the alternating copolymerization of $(R)$-limonene oxide and $\mathrm{CO}_{2}$ by using density functional theory (DFT) methods at the B97D3/6-311G*/LANL2DZ level of theory. Both initiation and propagation reactions comprising the Al-catalyst 2 were studied as described below giving important and unique insight in the chemo and stereoselectivities observed experimentally.

Initiation reaction. The ring opening of the trans $(\mathbf{A})$ and cis $(R)$-limonene oxide $(\mathbf{B})$ was first evaluated in the presence of chloride as well as bromide nucleophiles. This step usually involves a concerted transition state (TS1 in Figure 3) which is characterized by the breaking of the $\mathrm{C}_{a \mathrm{p}}-\mathrm{O}$ epoxide bond and the simultaneous formation of a $\mathrm{C}_{a \mathrm{n}}-\mathrm{Cl} / \mathrm{Br}$, leading to the formation of an alkoxide intermediate (Int-1 in Figure 3). ${ }^{[6,11 a]}$ The nucleophilic attack can occur at the $\alpha$ carbon (most substituted carbon) or the $\beta$ carbon (least substituted carbon) atoms of the cis/trans limonene oxide, and therefore eight possible ways of epoxide ring opening should be considered. The fourth and fifth columns of Table 3 collect the activation free-energies calculated for this step, once the epoxide is activated by the Lewis acidic aluminum center in complex 2 .

\begin{tabular}{|c|c|c|c|c|}
\hline \multirow[t]{2}{*}{ Substrate } & \multirow[t]{2}{*}{ Carbon } & \multirow[t]{2}{*}{$\begin{array}{c}\text { NBO population } \\
\text { analysis }^{[a]}\end{array}$} & \multicolumn{2}{|c|}{ 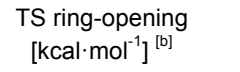 } \\
\hline & & & $\mathrm{Cl}$ & $\mathrm{Br}$ \\
\hline & $\alpha$ & $0.27(0.31)$ & 2.8 & 8.9 \\
\hline & $\beta$ & $0.10(0.12)$ & 4.7 & 10.2 \\
\hline B. & $\alpha$ & $0.27(0.31)$ & 0.7 & 6.1 \\
\hline & $\beta$ & $0.11(0.13)$ & 3.8 & 9.1 \\
\hline
\end{tabular}

[a] The first value corresponds to the charge obtained for isolated epoxide; whereas the second (in parenthesis), for the activated substrate due to coordination with the Al-catalyst 2. [b] Ring-opening barriers calculated from the energy difference between the transition state $\mathrm{TS}$ and initial complex coordination. $\mathrm{Cl}$ and $\mathrm{Br}$ were used as a nucleophilic reagent.

In general, barriers for the chloride attack are much lower than those obtained for the bromide attack. In all the cases, the $\alpha$ attack is favoured over the $\beta$ one, as supported by the NBO population analysis included also in Table 3 . It is commonly thought that the most substituted $\alpha$ carbon is less reactive than the least hindered because of the electronic effects induced by the methyl group. In contrast, the $\alpha$ attack was found to be more feasible, with the cis conformation being the preferred way over the trans substrate by $2.1 \mathrm{kcal} \cdot \mathrm{mol}^{-1}$. Alternatively, for the $\beta$ attack, this energy difference is less marked $\left(0.9 \mathrm{kcal} \cdot \mathrm{mol}^{-1}\right)$ although the cis isomer still remains favored. Because of the better results obtained using the nucleophilic chloride, this species was selected as co-catalyst for the alternating copolymerization, and thus decreasing the number of possible pathways to study. Figure 3 illustrates the free-energy profiles for the Al-complex 2 /chloride catalyzed initiation of the copolymerization of cis/trans- $(R)$-limonene oxide 


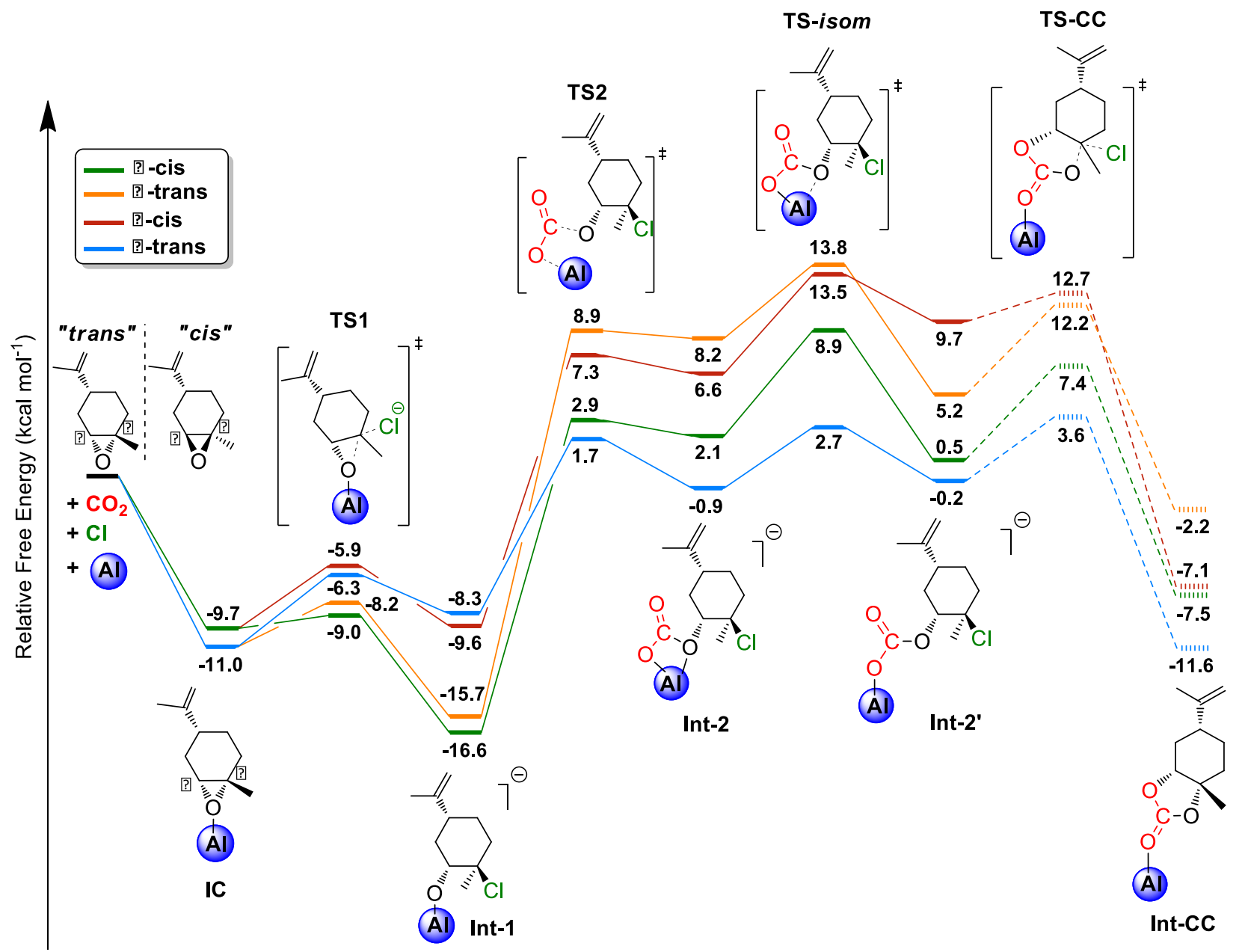

Figure 3. Free-energy profiles for the initiation reaction of copolymerization between cis/trans-( $R$-limonene oxide and $\mathrm{CO}_{2}$ catalyzed by the $\mathrm{Al}$-complex $2 /$ chloride binary system, and considering nucleophilic attack on the $\alpha$ and $\beta$ positions resulting in four different pathways. As solvent, 1 -hexanol was evaluated at $25{ }^{\circ} \mathrm{C}$. Barriers for the backbiting reactions appear in dashed lines. Note that only the pathway involving the trans substrate $\mathbf{A}$ ( $\alpha$ attack) is visualized with accompanying schematic structures.

and $\mathrm{CO}_{2}$, taking into account the nucleophilic attack by chloride on the $\alpha$ and $\beta$ positions of both epoxides. The starting point is the assembly of the isolated reactants, i.e., the Al-complex 2, chloride, cis/trans epoxide and $\mathrm{CO}_{2}$ for which the total free energy is set to $0.0 \mathrm{kcal} \cdot \mathrm{mol}^{-1}$. Then, the catalytic cycle begins with the coordination of each epoxide to the $\mathrm{Al}(\mathrm{III})$ center of complex 2 (allowing for epoxide activation) yielding two different complexes IC. This process is exergonic by $9.7 \mathrm{kcal} \cdot \mathrm{mol}^{-1}$ for the cis-coordinated (trace in green) complex and $11.0 \mathrm{kcal} \cdot \mathrm{mol}^{-1}$ for the trans one (trace in blue), showing thus a (slight) preference towards formation of the latter complex.

As indicated above, the ring-opening step leads to formation of the metal-alkoxide Int-1. It can be observed that the intermediates obtained by nucleophilic attack on the $\alpha$ position are energetically more stable than those involving the $\beta$ attack; the $\alpha$ carbon is more electrophilic than the $\beta$ one, as explained before by the NBO population analysis (Table 3 ). It is worth noting that the $\alpha$ carbon is a stereogenic center; consequently the nucleophilic attack of chloride on this carbon center evolves with inversion of configuration. Thus, the cis-coordinated epoxide evolves into the most stable trans-product Int-1 (trace in green) and the trans one is converted into the cis metal-alkoxide intermediate (trace in orange). For the $\beta$ attack, retention of configuration holds since the nucleophilic attack does not involve a stereogenic center.

Following the epoxide ring opening by the nucleophilic chloride, $\mathrm{CO}_{2}$ is inserted into the Al-O alkoxide bond of Int-1 via transition state TS2. The intermolecular $\mathrm{CO}_{2}$ insertion was located to be less energetically demanding for the $\beta$-trans and $\alpha$-cis pathways, with relative barriers of 1.7 and $2.9 \mathrm{kcal} \cdot \mathrm{mol}^{-1}$, respectively. The highest barrier was calculated for the $\alpha$-trans pathway, having an activation energy of $24.6 \mathrm{kcal} \cdot \mathrm{mol}^{-1}$. This step affords the hemi-carbonates Int-2, which are coordinated by two oxygen atoms to the $\mathrm{Al}(\mathrm{III})$ center. These intermediates follow the same stability trends as the preceding TS2, and could suffer isomerization (through TS- 
isom) to form the linear hemi-carbonate Int-2'. In this case, the $\beta$-trans and a-cis pathways still lead to the most stable intermediates. The isomerization of Int-2 


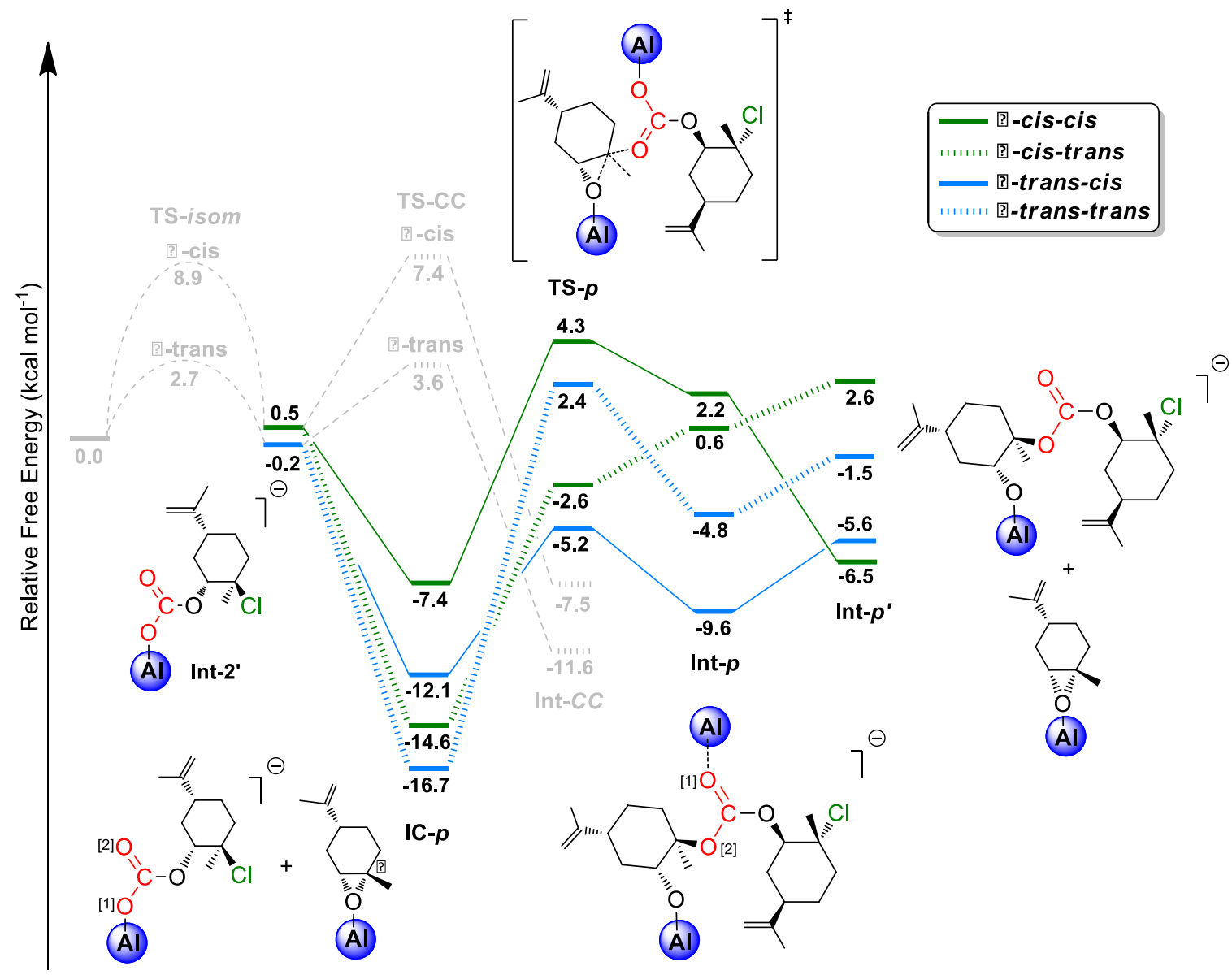

Figure 4. Free-energy profiles for the propagation of the bimetallic copolymerization between $\mathrm{CO}_{2}$ and cis/trans- $(R)$-limonene oxide mediated by the Al-complex 2 /chloride binary system, and considering the nucleophilic attack by the carbonyl oxygen (O[2]) on the $\alpha$ carbon of the epoxides. As solvent, 1-hexanol was evaluated at $25{ }^{\circ} \mathrm{C}$. The barriers for the isomerization and the backbiting reactions of the initiation process appear in grey dashed lines. Note that only the pathway involving the trans substrate $\mathbf{A}$ ( $\alpha$ attack) is visualized with accompanying schematic structures (i.e., formation of a cis-cis dimer).

is rate-determining for the $\alpha$-trans, $\beta$-cis and $\alpha$-cis profiles, with activation barriers (calculated from the alkoxide intermediate Int-1) of $29.5,23.1$ and $25.5 \mathrm{kcal} \cdot \mathrm{mol}^{-1}$, respectively. In the case of the $\beta$-trans profile, this is valid if the reaction is evaluated only until formation of the intermediate Int-2' (rather than taking into account the subsequent backbiting reaction as further discussed below) involving an activation barrier of $13.7 \mathrm{kcal} \cdot \mathrm{mol}^{-1}$, which is calculated from the most stable complex IC.

Having reached this point, two possible routes can be followed by these intermediates; the backbiting of the linear hemicarbonate Int-2' yielding the (undesired) cyclic carbonate, or consecutive addition of new epoxide and $\mathrm{CO}_{2}$ monomers allowing for propagation towards an alternating copolymer. The backbiting reaction is shown in Figure 3 and goes through TS-CC. The latter displays features of a classical $S_{N} 2$ type transition state similar to the epoxide ring opening with intramolecular ring-closing and concomitant release of the chloride nucleophile. This step requires slightly lower barriers than that involved in the isomerization reaction, with the $\beta$-trans profile being the only exception with relative barrier of $3.6 \mathrm{kcal} \cdot \mathrm{mol}^{-1}$. The alternating propagation reaction is separately discussed in the next section and will explain why there is a preference for polycarbonate formation from limonene oxide and $\mathrm{CO}_{2}$ using $\mathrm{Al}$ complex 2/PPNCl as catalyst system.

\section{Propagation reaction.}

Once the linear hemi-carbonate Int-2' is formed in the initiation process, several attack routes can be followed. ${ }^{[18]}$ The carbonyl oxygen of the four resulting Int-2' species serve as nucleophiles for attacking two different epoxide conformations (cis or trans) on two different carbon atoms $(\alpha$ or $\beta$ ). This situation generates sixteen possible profiles to investigate (see Scheme $S 1$ in the Supporting Information). In order to decrease the computational efforts, we decided to study the most feasible pathways, based on the outcome from the initiation reaction. Thus, the number of pathways was reduced to four by considering the attack by the most 
stable $\alpha$-cis and $\beta$-trans hemi-carbonates on the $\alpha$ carbon (the most electrophilic atom) of the cis and trans epoxides. The free-energy profiles for the alternating propagation step of cis/trans- $(R)$-limonene oxide and $\mathrm{CO}_{2}$, taking into account the previous considerations, are illustrated in Figure 4.

The propagation process requires two aluminum centers (bimetallic mechanism). ${ }^{[17,19]}$ It can be firstly observed the formation of a very stable adduct between the intermediate Int-2' and the complex having a new epoxide substrate coordinated to another Alcomplex 2 (IC-p). Natural bond orbital (NBO) population analysis on this complex shows small difference in the value of the charge assigned to the oxygen atoms of the carbonate Int-2'. The oxygen atom labeled O[1] bound to the Al center in Figure 4 exhibits a charge of -0.83 ; whereas for the carbonyl oxygen (O[2]), a value of -0.69 was obtained. Although the oxygen $O[1]$ is slightly more nucleophilic than the carbonyl oxygen $\mathrm{O}[2]$, reaction progress through $\mathrm{O}[1]$ leads to the higher barriers for subsequent steps of the free-energy profile (see Figure S39 in the Supporting Information). In all cases, a substantial lower energy between the formed IC-p and the transition state of the backbiting reaction (TS-CC; Figure 3 and grey traces in Figure 4) was found. Thus, for instance, the resulting complexes between the a-cis Int-2' and the coordinated cis and trans epoxides (i.e., a-cis-cis and a-cis-trans dimers in Figure 4) were found to be more stable than their corresponding TS-CC by 14.8 and $22.0 \mathrm{kcal} \cdot \mathrm{mol}^{-1}$, respectively. In the case of the $\beta$-trans carbonate Int-2' forming an initial adduct with each (coordinated) substrate attached to a second Al complex 2, this energy difference becomes $15.7 \mathrm{kcal} \cdot \mathrm{mol}^{-1}$ combining with the cis-epoxide and $20.3 \mathrm{kcal} \cdot \mathrm{mol}-1$ with the trans one that would result in dimers a-trans-cis and a-trans-trans.

Following the reaction coordinate, the next step is the epoxide ring opening, which is undertaken by nucleophilic attack of the carbonyl oxygen labeled O[2] (in Figure 4) on the most substituted carbon ( $\alpha$ ) of each epoxide isomer bound to one of the $\mathrm{Al}(\mathrm{III})$ centers in IC-p. Similar as for the initiation process, in the propagation reaction the epoxide ring-opening is characterized by a concerted transition state TS-p. The $\alpha$-cis-cis profile shows the highest activation barrier being $20.9 \mathrm{kcal} \cdot \mathrm{mol}^{-1}$ (having a relative energy of $4.3 \mathrm{kcal} \cdot \mathrm{mol}^{-1}$ ), calculated from the most stable intermediate of the initiation process Int-1 with a relative energy of -16.6 $\mathrm{kcal} \cdot \mathrm{mol}^{-1}$ (Figure 3). In contrast, the TS-p for the $\beta$-trans-cis pathway involves an activation barrier of only $6.9 \mathrm{kcal} \cdot \mathrm{mol}^{-1}$ (estimated from the adduct IC-p). In the case of the $\beta$-trans-trans and $\alpha$-cis-trans profiles, these barriers were obtained in a similar way as described for the previous pathways, and lead to values of 19.1 and $14.0 \mathrm{kcal} \cdot \mathrm{mol}^{-1}$, respectively.

Once passing through the barrier for TS-p, the formation of the intermediate Int-p occurs which has both Al complexes still coordinated. However, the strength of interaction between the oxygen from the alkoxide and the Al center is much stronger than that observed for the oxygen $\mathrm{O}$ [1] of the coordinated carbonate and the $\mathrm{Al}(\mathrm{III})$ center from Int-2'. Hence, it is proposed that Int-p can evolve into intermediate Int-p' by releasing the Al complex from the carbonate and allowing for coordination of a new trans- $(R)$ limonene oxide monomer. This reaction is endergonic by $2.6 \mathrm{kcal} \cdot \mathrm{mol}^{-1}$ for the $\alpha$-cis-trans profile. The remaining processes are slightly exergonic, with a release of $-1.5,-5.6$ and $-6.5 \mathrm{kcal} \cdot \mathrm{mol}^{-1}$ in the case of the $\beta$-trans-trans, $\beta$-trans-cis and $\alpha$-cis-cis pathways, respectively. Interestingly, both the energetically most stable dimeric units Int-p' resulting from the $\beta$-trans-cis and $\alpha$-cis-cis profiles will contain merely trans units in their backbone ${ }^{[17]}$ which is overall well in line with the experimental results. The current catalytic process based on Al complex 2/PPNCl shows two main features: (1) a clear preference for the faster conversion of cis-limonene oxide (B), and (2) the resulting copolymers contain a significant higher amount of trans versus cis units (up to 98:2, Table 2) where the use of pure cis-limonene oxide will result in the formation of a nearly stereo-regular all-trans polycarbonate.

\section{Conclusions}

This work showcases the rare but efficient conversion of a tri-substituted oxirane (limonene oxide) and $\mathrm{CO}_{2}$ into a $100 \%$ bio-based polycarbonate using an $\mathrm{Al}(\mathrm{III})$ amino-trisphenolate/PPNCl binary catalyst under mild reaction conditions $\left(42^{\circ} \mathrm{C}, 10\right.$ bar). The typical features of this process involve a catalyst system based on an earth-abundant metal and modular, cheap and easily accessible ligand systems. The catalyst is highly robust as testified by the high conversion levels that can be attained $(>70 \%)$ of the limonene oxide under neat reaction conditions. The poly(limonene)carbonates can be produced in a stereo-regular fashion (when pure cislimonene oxide is used) and its copolymer exhibits a $T_{\mathrm{g}}$ value of around $112^{\circ} \mathrm{C}$ potentially useful in the context of finding bioalternatives for commercially produced polycarbonates. The DFT results show unique and important insight into the chemo- and stereo-selectivity of the limonene oxide/ $\mathrm{CO}_{2}$ coupling reaction mediated by $\mathrm{Al}$ complex 2/PPNCl. This information is highly valuable to develop other types of bio-based poly(carbonates) and/or polyesters. These types of bio-based polymers are expected to grow significantly in importance in the forthcoming development of new, sustainable alternative thermoplastics for the specialty polymer industries. Taking into account the favorable characteristics of the presented $\mathrm{Al}(\mathrm{III})$ amino-triphenolate complex and their reactivity and robustness, we are currently planning to widen the scope of accessible $\mathrm{CO}_{2}$-based polymers.

\section{Experimental Section}

General materials and methods 
All solvents, reagents, and chemicals were purchased from commercial suppliers and used as received unless noted otherwise. Carbon dioxide was purchased from PRAXAIR and used without further purification. The iron and aluminium(III) amino triphenolate complexes 1-4 were prepared following previously reported protocols. ${ }^{[8,9,11]} \mathrm{PPNI}, \mathrm{PPNBr}$ and $\mathrm{PPNCl}$ were prepared as previously described. ${ }^{[20]} \mathrm{NMR}$ spectra were recorded on a Bruker AV400 or AV-500 spectrometer and referenced to the residual NMR solvent signals. Mass spectrometric analyses were performed by the Research Support Group at the ICIQ. Gel Permeation Chromatography (GPC) measurements were carried out externally at the Laboratoire de Chimie et Procédés de Polymérisation (LCCP) in Lyon (France) and reported $M_{n}$ values and polydispersities were determined against a series of discrete PS standards. Differential Scanning Calorimetry (DSC) and Thermogravimetric Analysis (TGA) were performed at ICIQ using a Mettler Toledo DSC822e and TGA/SDTA851 machine, respectively.

\section{Copolymerization reactions}

Typical conditions: $(R)$-limonene oxide $(1.0 \mathrm{~g}, 6.57 \mathrm{mmol})$, the Al catalyst $(0.5-1.0 \mathrm{~mol} \%)$ and co-catalyst (the respective chloride or bromide, $0.25-2.5$ $\mathrm{mol} \%$ ) were placed in a $30 \mathrm{~mL}$ stainless steel reactor with a stirring bar. Three cycles of pressurization and depressurization with carbon dioxide were applied $\left(\mathrm{pCO}_{2}=5 \mathrm{Bar}\right)$ before finally stabilizing the pressure at 10 bar. The reactor was then heated to the required temperature for the chosen time. After that, the reactor was cooled down to room temperature before depressurizing. After this time, the conversion and selectivity was calculated using ${ }^{1} \mathrm{H}$ NMR analysis $\left(\mathrm{CDCl}_{3}\right)$ of an aliquot of the reaction mixture. The starting material was removed from the reaction mixture in vacuo. The poly(limonene)carbonates were further purified dissolving the reaction mixture in a small amount of dichloromethane followed by precipitation of the products by addition of acidic methanol $(\mathrm{HCl} 1 \mathrm{M}$ in methanol). After that, the polymer was filtered and dried in vacuo followed by analysis by, depending on the sample, a combination of NMR, MALDI-TOF, GPC, TGA and DSC. Reactions under more rigorously dry conditions were conducted in a Fischer-Porter reactor (see Supporting Information). Typically, $(R)$-limonene oxide (cis and trans mix-ture) and pure cis-(R)-limonene oxide were each distilled from calcium hydride under reduced pressure following three cycles (30 min each) of vacuum- $\mathrm{N}_{2}$ purging, and stored in a glove box. The catalyst and co-catalyst were dried by three cycles (30 min each) of vacuum- $\mathrm{N}_{2}$ purging in a silicon bath at $70^{\circ} \mathrm{C}$. The Fisher-Porter reactor was filled inside the glove box and closed. After one cycle of pressurization and depressurization with carbon dioxide $\left(p \mathrm{CO}_{2}=3 \mathrm{Bar}\right)$ the reactor was pressurized at $5 \mathrm{Bar}$ and heated in a silicon bath at $45^{\circ} \mathrm{C}$ for the chosen time. The reaction mixture was dissolved in dichloromethane and the poly(limonene)carbonate was precipitated with acidic methanol ( $\mathrm{HCl} 1 \mathrm{M}$ in methanol). Analysis was done as reported for the other poly(carbonate) samples.

\section{Computational details}

All calculations in this study were carried out using the Gaussian 09 package. ${ }^{[21]}$ The B97D3 functional was employed, which includes empirical dispersion energy corrections as introduced by Grimme. ${ }^{[22]}$ The standard $6-311 \mathrm{G}(\mathrm{d}, \mathrm{p})$ basis set was used to describe the $\mathrm{H}$, $\mathrm{C}$, $\mathrm{N}$ and $\mathrm{O}$ atoms. The relativistic effective core pseudo potential LANL2DZ was used for $\mathrm{Al}, \mathrm{Br}$ and $\mathrm{Cl}$ atoms together with its associated basis set. Full geometry optimizations were performed without constrains. The nature of the stationary points encountered was characterized either as minima or transition states by means of harmonic vibrational frequencies analysis. Gibbs free energies were calculated at standard conditions $(T=298 \mathrm{~K}, \mathrm{p}=1 \mathrm{bar})$. In order to introduce sol-vent effects, single point calculations were performed on the gas-phase optimized structures by using the polarizable continuum model (PCM). The dielectric constant $(\varepsilon)$ of the polarizable medium was set to the value reported for the simplest epoxide, ethylene oxide $(\varepsilon=12.42)^{[23]}$ as the reaction takes place in the limonene oxide rich phase. The 1-hexanol solvent was used for this purpose $(\varepsilon=12.51)$, as implemented in Gaussian.

\section{Acknowledgements}

We thank ICIQ, ICREA and the Spanish Ministerio de Economía y Competitividad (MINECO) through projects CTQ-2011-27385, CTQ2011-29054-C02-02, the Severo Ochoa Excellence Accreditation 2014-2018 (SEV-2013-0319), and FPI fellowships to L.P.C. and F. C. G. We also thank AGAUR for support through 2009-SGR-259. Dr. Noemí Cabello, Sofía Arnal and Vanessa Martínez are acknowledged for the MALDI-TOF MS analyses.

Keywords: Al catalysis $\cdot \mathrm{DFT} \cdot$ limonene oxide $\bullet$ polycarbonates $\bullet$ stereoregularity

[1] a) R. A. Sheldon, Green Chem. 2014, 16, 950-963; b) M. Poliakoff, P. T. Anastas, Nature 2001, 413, 257-257; c) M. Poliakoff, J. M. Fitzpatrick, T. R. Farren, P. T. Anastas, Science 2002, 297, 807-810; d) R. Ciriminna, M. Lomeli-Rodriguez, P. Demma Carà, J. A. Lopez-Sanchez, M. Pagliaro, Chem. Commun. 2014, 50, 15288-15296; e) M. Winkler, C. Romain, M. A. R. Meier, C. K. Williams, Green Chem. 2015, DOI: 10.1039/C4GC01353K.

[2] For some recent reviews: a) M. Cokoja, C. Bruckmeier, B. Rieger, W. A. Herrmann, F. E. Kühn, Angew. Chem. Int. Ed. 2011, 50, 8510-8537; b) A Decortes, A. M. Castilla, A. W. Kleij, Angew. Chem. Int. Ed. 2010, 49, 9822-9837; c) N. Kielland, C. J. Whiteoak, A. W. Kleij, Adv. Synth. Catal. 2013, 355, 2115-2138; d) M. Mikkelsen, M. Jørgensen, F. C. Krebs, Energy Environ. Sci. 2010, 3, 43-81; e) M. Aresta, A. Dibenedetto, A. Angelini, Chem. Rev. 2014 114, 1709-1742; f) Y. Tsuji, T. Fujihara, Chem. Commun. 2012, 48, 9956-9964; g) H. Maeda, Y. Miyazaki, T. Ema, Catal. Sci. Technol. $2014,4,1482-1497$.

[3] For reviews on this topic: a) M. R. Kember, A. Buchard, C. K. Williams, Chem. Commun. 2011, 47, 141-163; b) D. J. Darensbourg, Chem. Rev. 2007, 107, 2388-2410; c) K. Nozaki, Pure Appl. Chem. 2004, 76, 541-546; d) X.-B. Lu, D. J. Darensbourg, Chem. Soc. Rev. 2012, 41, 1462-1484; e) G. W. Coates, D. R. Moore, Angew. Chem. Int. Ed. 2004, 43, 6618-6639; f) S. Klaus, M. W. Lehenmeier, C. E. Anderson, B. Rieger, Coord. Chem. Rev. 2011, 255, 14601479; g) X.-B. Lu, W.-M. Ren, G.-P. Wu, Acc. Chem. Res. 2012, 45, 1721-1735.

[4] Selected original contributions: a) C. T. Cohen, T. Chu, G. W. Coates, J. Am. Chem. Soc. 2005, 127, 10869-10878; b) M. R. Kember, P. D. Knight, P. T. R. Reung, C. K. Williams, Angew. Chem. Int. Ed. 2009, 48, 931-933; c) D. J. Darensbourg, S. J. Wilson, J. Am. Chem. Soc. 2011, 133, 18610-18613; d) K. Nozaki, K. Nakano, T. Hiyama, J. Am. Chem. Soc. 1999, 121, 11008-11009; e) K. Nakano, T. Kamada, K. Nozaki, Angew. Chem. Int. Ed. 2006, 45, 7274- 
7277; f) X.-B. Lu, L. Shi, Y.-M. Wang, R. Zhang, Y.-J. Zhang, X.-J. Peng, Z.-C. Zhang, B. Li, J. Am. Chem. Soc. 2006, 128, 1664-1674; g) G.-P. Wu, S.-H. Wei, W.-M. Ren, X.-B. Lu, T.-Q. Xu, D. J. Darensbourg, J. Am. Chem. Soc. 2011, 133, 15191-15199; h) S. I. Vagin, R. Reichardt, S. Klaus, B. J. Rieger, J. Am. Chem. Soc. 2010, 132, 14367-14369; i) E. K. Noh, S. J. Na, S. Sujith, S. W. Kim, B. Y. Lee, J. Am. Chem. Soc. 2007, 129, 8082-8083; j) Y. Liu, M. Wang, W.-M. Ren, K.-K. He, Y.-C. Xu, J. Liu, X.-B. Lu, Macromolecules 2014, 47, 1269-1276; k) F. Jutz, A. Buchard, M. R. Kember, S. Bodil Fredriksen, C. K. Williams, J. Am. Chem. Soc. 2011, 133, 17395-17405.

[5] a) C. M. Byrne, S. D. Allen, E. B. Lobkovsky, G. W. Coates, J. Am. Chem. Soc. 2004, 126, 11404-11405; b) F. Auriemma, C. de Rosa, M. Rosaria Di Caprio, R. di Girolamo, W. Chadwick Ellis, G. W. Coates, Angew. Chem. Int. Ed. 2014, DOI: 10.1002/anie.201410211.

[6] F. Castro-Gómez, G. Salassa, A. W. Kleij, C. Bo, Chem. Eur. J. 2013, 19, 6289-6298.

[7] a) C. Beattie, M. North, P. Villuendas, C. Young, J. Org. Chem. 2013, 78, 419-426; b) W. J. Kruper, D. V. Dellar, J. Org. Chem. 1996, 60, 725-727; c) M. Bähr, A. Bitto, R. Mülhaupt, Green Chem. 2012, 14, 1447-1454; d) J. Wu, J. A. Kozak, F. Simeon, T. A. Hatton, T. F. Jamison, Chem. Sci. 2014, 5, 12271231; e) J. Qin, P. Wang, Q. Li, Y. Zhang, D. Yuan, Y. Yao, Chem. Commun. 2014, 50, 10952-10955; f) T. Ema, Y. Miyazaki, J. Shimonishi, C. Maeda, J.Y. Hasegawa, J. Am. Chem. Soc. 2014, 136, 15270-15279.

[8] a) C. J. Whiteoak, E. Martin, E. C. Escudero-Adán, A. W. Kleij, Adv. Synth. Catal. 2013, 355, 2233-2239; b) C. J. Whiteoak, E. Martin, M. Martínez Belmonte, J. Benet-Buchholz, A. W. Kleij, Adv. Synth. Catal. 2012, 354, 469-476; c) C. J. Whiteoak, B. Gjoka, E. Martin, M. Martínez Belmonte, E. C. Escudero-Adán, C. Zonta, G. Licini, A. W. Kleij, Inorg. Chem. 2012, 51, 10639-10649.

[9] C. J. Whiteoak, N. Kielland, V. Laserna, E. C. Escudero-Adán, E. Martin, A. W. Kleij, J. Am. Chem. Soc. 2013, 135, $1228-1231$.

[10] C. J. Whiteoak, A. H. Henseler, C. Ayats, A. W. Kleij, M. A. Pericàs, Green Chem. 2014, 16, 1552-1559.

[11] a) C. J. Whiteoak, N. Kielland, V. Laserna, F. Castro-Gómez, E. Martin, E. C. Escudero-Adán, C. Bo, A. W. Kleij, Chem. Eur. J. 2014, 20, 2264-2275; b) V. Laserna, G. Fiorani, C. J. Whiteoak, E. Martin, E. Escudero-Adán, A. W. Kleij, Angew. Chem. Int. Ed. 2014, 53, $10416-10419$.

[12] M. Taherimehr, S. M. Al-Amsyar, C. J. Whiteoak, A. W. Kleij, P. P. Pescarmona, Green Chem. 2013, 15, 3083-3090.

[13] Please note that the lower conversion of the limonene oxide at higher temperatures is not likely an effect of catalyst decomposition. Previously we reported that $\mathrm{Al}$ (amino-triphenolate) based catalyst systems can be easily operated at temperatures up to $90^{\circ} \mathrm{C}$ without signs of decomposition and maintaining excellent activity for organic carbonate formation, see ref. 9 and 11.

[14] M. R. Kember, C. K. Williams, J. Am. Chem. Soc. 2012, 134, 15676-15679.

[15] The influence of the stirring technique suggests that the increasing viscosity of the mixtures observed during the course of the copolymerization reaction (in particular at high conversion levels) strongly affects the mass transfer properties.

[16] The reported DSC data refer to the second heating run, see also the Supporting Information.

[17] A monometallic mechanism was also investigated for the alternating copolymerization of $\mathrm{CO}_{2}$ and epoxides, affording higher free-energy barriers for the propagation reaction. A manuscript including those results is in preparation. See also: K. Nakano, K. Nozaki, T. Hiyama, J. Am. Chem. Soc. 2003, 125, 5501-5510.

[18] Note that the formulation cis-cis (for instance) for a dimeric carbonate unit refers to initial conversion of a cis-limonene oxide monomer followed by the insertion (through an $\mathrm{S}_{\mathrm{N}} 2$ like pathway, TS-p in Figure 4) of a second cis-limonene oxide monomer to afford a trans-trans dimeric carbonate unit. Alternatively, the conversion of two trans limonene oxide monomers in a similar way also leads to a trans-trans dimeric carbonate intermediate (cf., Int-p' in Figure 4).

[19] Williams et al. have recently described a bimetallic $\mathrm{Zn}$ catalyst that is active in $\mathrm{CO}_{2} / \mathrm{CHO}$ copolymerization, and computational efforts have also demonstrated the necessity of two metal centres for efficient copolymerization, see: A. Buchard, F. Jutz, M. R. Kember, A. J. P. White, H. S. Rzepa, C. K. Williams, Macromolecules 2012, 45, 6781-6795.

[20] F. A. L’Epplatenier, A. Pugin, Helv. Chim. Acta 1975, 58, 917-923.

[21] Gaussian, Revision D.01, M. J. Frisch, G. W. Trucks, H. B. Schlegel, G. E. Scuseria, M. A. Robb, J. R. Cheeseman, G. Scalmani, V. Barone, B. Mennucci, G. A. Petersson, H. Nakatsuji, M. Caricato, X. Li, H. P. Hratchian, A. F. Izmaylov, J. Bloino, G. Zheng, J. L. Sonnenberg, M. Hada, M. Ehara, K. Toyota, R. Fukuda, J. Hasegawa, M. Ishida, T. Nakajima, Y. Honda, O. Kitao, H. Nakai, T. Vreven, J. A. Montgomery, Jr., J. E. Peralta, F. Ogliaro, M. Bearpark, J. J. Heyd, E. Brothers, K. N. Kudin, V. N. Staroverov, R. Kobayashi, J. Normand, K. Raghavachari, A. Rendell, J. C. Burant, S. S. Iyengar, J. Tomasi, M. Cossi, N. Rega, N. J. Millam, M. Klene, J. E. Knox, J. B. Cross, V. Bakken, C. Adamo, J. Jaramillo, R. Gomperts, R. E. Stratmann, O. Yazyev, A. J. Austin, R. Cammi, C. Pomelli, J. W. Ochterski, R. L. Martin, K. Morokuma, V. G. Zakrzewski, G. A. Voth, P. Salvador, J. J. Dannenberg, S. Dapprich, A. D. Daniels, Ö. Farkas, J. B. Foresman, J. V. Ortiz, J. Cioslowski and D. J. Fox, 2013, Gaussian, Inc., Wallingford CT.

[22] a) S. Grimme, J. Comp. Chem. 2006, 27, 1787-1799; b) S. Grimme, S. Ehrlich, L. Goerigk, J. Comp. Chem. 2011, 32, 1456-1465.

[23] CRC Handbook of Chemistry and Physics, D.R. Lide (ed), 84th Ed. CRC Press LLC, Florida 2003. 


\section{Entry for the Table of Contents:}

\section{FULL PAPER}

The sporadic but efficient copolymerization of $(R)$ limonene oxide and $\mathrm{CO}_{2}$ into a bio-renewable polycarbonate is reported. The process is Al-catalysed showing higher preference for the conversion of the cis-substrate. The stereo-regular features and the preference for the formation of a virtually all trans-copolymer have been examined in detail using DFT analysis. renewable monomer
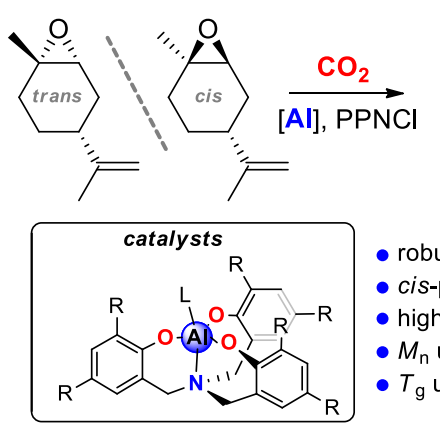

a biopolymer

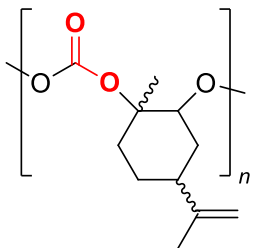

- robust catalyst

- cis-preference

- high stereoregularity

- $M_{\mathrm{n}}$ up to $10.6 \mathrm{~kg} / \mathrm{mol}$

- $T_{\mathrm{g}}$ up to $112^{\circ} \mathrm{C}$
Leticia Peña Carrodeguas, Fernando Castro-Gómez, Joan González-Fabra, Carles Bo*and Arjan W. Kleij*

Page No. - Page No.

Al(III) Catalyzed Formation of Poly(limonene)carbonate: DFT Analysis of the Origin of Stereoregularity 